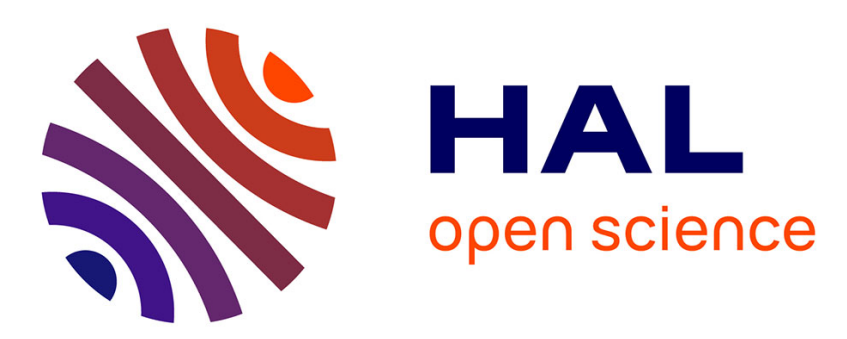

\title{
Facial non-repetitive edge-colouring of plane graphs
}

Frédéric Havet, Stanislav Jendrol', Roman Sotak, Erika Skrabulakova

\section{To cite this version:}

Frédéric Havet, Stanislav Jendrol', Roman Sotak, Erika Skrabulakova. Facial non-repetitive edgecolouring of plane graphs. Journal of Graph Theory, 2011, 66 (1), pp.38-48. 10.1002/jgt.20488 . inria-00638439

\section{HAL Id: inria-00638439 \\ https://hal.inria.fr/inria-00638439}

Submitted on 23 Oct 2016

HAL is a multi-disciplinary open access archive for the deposit and dissemination of scientific research documents, whether they are published or not. The documents may come from teaching and research institutions in France or abroad, or from public or private research centers.
L'archive ouverte pluridisciplinaire HAL, est destinée au dépôt et à la diffusion de documents scientifiques de niveau recherche, publiés ou non, émanant des établissements d'enseignement et de recherche français ou étrangers, des laboratoires publics ou privés. 


\title{
Facial non-repetitive edge-colouring of plane graphs
}

\author{
Frédéric Havet * Stanislav Jendrol’ ${ }^{\ddagger} \quad$ Roman Soták ${ }^{\ddagger}$ \\ Erika Škrabul'áková $\ddagger$
}

March 2, 2009

\begin{abstract}
A sequence $r_{1}, r_{2}, \ldots, r_{2 n}$ such that $r_{i}=r_{n+i}$ for all $1 \leq i \leq n$, is called a repetition. A sequence $S$ is called non-repetitive if no block (i.e. subsequence of consecutive terms of $S$ ) is a repetition. Let $G$ be a graph whose edges are coloured. A trail is called non-repetitive if the sequence of colours of its edges is non-repetitive. If $G$ is a plane graph, a facial nonrepetitive edge-colouring of $G$ is an edge-colouring such that any facial trail (i.e. trail of consecutive edges on the boundary walk of a face) is nonrepetitive. We denote $\pi_{f}^{\prime}(G)$ the minimum number of colours of a facial non-repetitive edge-colouring of $G$. In this paper, we show that $\pi_{f}^{\prime}(G) \leq 8$ for any plane graph $G$. We also get better upper bounds for $\pi_{f}^{\prime}(G)$ in the cases when $G$ is a tree, a plane triangulation, a simple 3-connected plane graph, a hamiltonian plane graph, an outerplanar graph or a Halin graph. The bound 4 for trees is tight.
\end{abstract}

\section{Introduction}

A sequence $r_{1}, r_{2}, \ldots, r_{2 n}$ such that $r_{i}=r_{n+i}$ for all $1 \leq i \leq n$, is called a repetition. A sequence $S$ is called non-repetitive if no block (i.e. subsequence of consecutive terms of $S$ ) is a repetition. The celebrated Thue Theorem [18] states that arbitrarily long non-repetitive sequences can be formed using only three symbols. Alon et al. [1] introduced a natural generalization of Thue's sequences for edge-colouring of graphs. An edge-colouring $\varphi$ of a graph $G$ is non-repetitive if the sequence of colours on any path in $G$ is non-repetitive. The Thue chromatic index of $G$, denoted $\pi^{\prime}(G)$, is the minimum number of colours of a non-repetitive edge-colouring of $G$. Thue's sequences show that the Thue

\footnotetext{
${ }^{*}$ Projet Mascotte, I3S (CNRS and University of Nice-Sophia Antipolis) and INRIA, 2004 Route des Lucioles, BP 93, 06902 Sophia-Antipolis Cedex, France. frederic.havet@sophia.inria.fr

¥Institute of Mathematics, Faculty of Science, P. J. Šafárik University, Jesenná 5, 04001 Košice, Slovakia. [stanislav.jendrol, roman.sotak, erika.skrabulakova] @upjs.sk
} 
chromatic index of a path is at most 3. Actually, $\pi^{\prime}\left(P_{n}\right)=3$, for all $n \geq 5$, as it is easy to see that any sequence of length 4 on two symbols contains a repetition. An immediate corollary is that the Thue chromatic index of a cycle is at most 4 . In [6], Currie showed that $\pi^{\prime}\left(C_{n}\right)=4$ only for $n \in\{5,7,9,10,14,17\}$. For other values of $n>5, \pi^{\prime}\left(C_{n}\right)=3$. Note that every non-repetitive edge-colouring is a proper edge-colouring. So for simple graphs $\pi^{\prime}(G) \geq \chi^{\prime}(G) \geq \Delta(G)$. Alon et al. [1] showed that $\pi^{\prime}(G) \leq c \Delta(G)^{2}$ for some absolute constant $c$ and conjecture that $\pi^{\prime}(G) \leq c^{\prime} \Delta(G)$ for some absolute constant $c^{\prime}$. They proved this conjecture for several classes of graphs: the complete graph $K_{n}$ on $n$ vertices satisfies $\pi^{\prime}\left(K_{n}\right) \leq 2 n$ and for any tree $T, \pi^{\prime}(T) \leq 4(\Delta(T)-1)$.

In this paper, we study a variant of non-repetitive edge-colouring for plane graphs which is less constrained. We use the standard terminology according to Bondy and Murty [3] except for few notations defined throughout this article. However we recall some frequently used terms.

Let $G$ be a plane graph. A facial trail in $G$ is a trail made of consecutive edges of the boundary walk of some face. A facial non-repetitive edge-colouring of $G$ is an edge-colouring of $G$ such that any facial trail is non-repetitive. The facial Thue chromatic index of $G$, denoted $\pi_{f}^{\prime}(G)$, is the minimum number of colours of a facial non-repetitive edge-colouring of $G$. Note that the facial Thue chromatic index depends on the embedding of the graph. For example, the graph depicted in Figure 1 has different facial Thue chromatic index depending on its embedding. With the embedding on the left, its facial Thue chromatic index is at least 4 as one of its face is a 5-cycle and with the embedding on the right its facial Thue chromatic index is 3 as shown by the depicted facial non-repetitive edge 3 -colouring. Note in addition that in general a facial non-
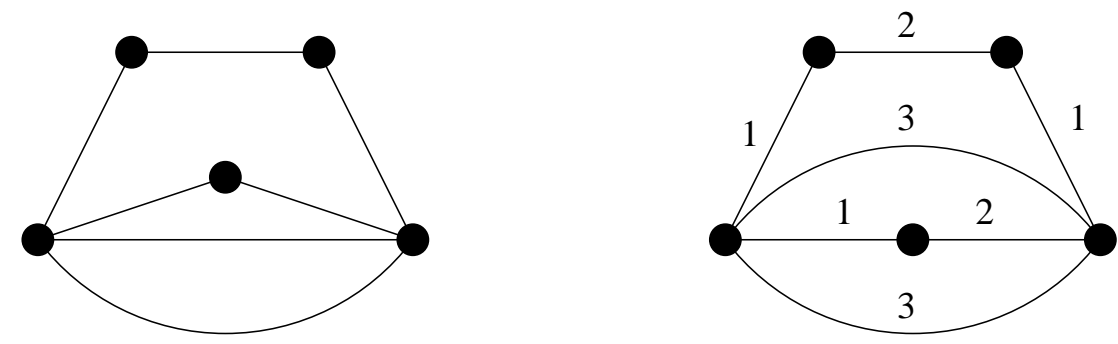

Figure 1: Two embeddings of the same graph with different facial Thue chromatic index

repetitive edge-colouring is not proper. See for example the facial non-repetitive 3 -edge-colouring of the graph on the right in Figure 1.

In the following, all the graphs we will consider come along with an embedding in the plane. The (geometric) dual of the plane graph $G=(V, E, F)$ is the graph $G^{*}=\left(V^{*}, E^{*}, F^{*}\right)$ with the following property: for each face $\alpha$ of $G$ there is a vertex $\alpha^{*}$ of $G^{*}$; two vertices $\alpha^{*}$ and $\beta^{*}$ are joined by an edge $e^{*}$ of $G^{*}$ if and only if they are incident to the same edge $e$ in $G$. The edge $e^{*}$ of $G^{*}$ is 
associated with the edge $e$ in $G$ and more generally the set of edges $X \subseteq E(G)$ is associated with $X^{*}=\left\{e^{*}: e \in X\right\}$. It is well known that the dual of $G^{*}$ is $G$ and that $G^{*}$ is also a plane graph.

Let $G$ be a 2-edge-connected plane graph. Then its dual $G^{*}$ is loopless. So a proper edge-colouring of $G^{*}$ exists. Let a proper edge-colouring of $G^{*}$ be given, then assigning to each edge $e$ of $G$ the colour of its corresponding edge in $G^{*}$ produces a facial non-repetitive edge-colouring of $G$. Hence the facial Thue chromatic index of $G$ is at most the chromatic index of its dual.

Proposition 1. Let $G$ be a 2-edge-connected plane graph. Then $\pi_{f}^{\prime}(G) \leq$ $\chi^{\prime}\left(G^{*}\right)$.

All the upper bounds on $\chi^{\prime}\left(G^{*}\right)$ applies to $\pi_{f}^{\prime}(G)$. Shannon's Theorem [16] implies $\pi_{f}^{\prime}(G) \leq\left\lceil\frac{3}{2} \Delta\left(G^{*}\right)\right\rceil$ and Vizing-Gupta Theorem [19,9] implies that $\pi_{f}^{\prime}(G) \leq \Delta\left(G^{*}\right)+\mu\left(G^{*}\right)$ where $\mu\left(G^{*}\right)$ is the maximum of the edges multiplicities in $G^{*}$. For some special classes of 2-edge-connected plane graphs $\chi^{\prime}\left(G^{*}\right)=$ $\pi_{f}^{\prime}(G)$. This is in particular the case for plane triangulations.

Corollary 2. Let $G$ be a plane triangulation. Then $\pi_{f}^{\prime}(G)=3$.

Proof. As $G$ is a triangulation, its dual $G^{*}$ is a 2-connected cubic plane graph. As shown by Tait in 1880 (see [17, 20]) the 3-edge-colourability of such graphs is equivalent to the Four Colour Theorem $([15,20])$. Hence, by Proposition 1, $\pi_{f}^{\prime}(G) \leq \chi^{\prime}\left(G^{*}\right) \leq 3$.

Now every face of $G$ is a triangle and requires at least 3 colours in a facial non-repetitive edge-colouring. So $\pi_{f}^{\prime}(G)=3$.

However the chromatic index of a general (plane) graph $G^{*}$ is not bounded as it is at least its maximum degree $\Delta\left(G^{*}\right)$. In the Section 4 , we show that the facial Thue chromatic index of a plane graph is bounded by 8 . We get tight upper bounds for trees (Section 3), simple 3-connected graphs and Halin graphs (Section 4). In the Section 5, we improve this upper bounds when $G$ is hamiltonian and thus derive bounds for outerplanar graphs. In the final Section 6 we discuss our results and formulate an open problem.

\section{Lemmas on Thue sequences}

To be able to prove the theorems below we need some preliminary lemmas.

For a sequence of symbols $S=a_{1}, a_{2}, \ldots, a_{n}$ with $a_{i} \in \mathbb{A}$, for all $1 \leq k \leq$ $l \leq n$, we denote by $S_{k, l}$ the block $a_{k}, a_{k+1}, \ldots, a_{l}$.

Lemma 3. Let $A=a_{1}, a_{2}, \ldots, a_{m}$ be a non-repetitive sequence with $a_{i} \in \mathbb{A}$ for all $i=1,2, \ldots, m$. Let $B^{i}=b_{1}^{i}, b_{2}^{i}, \ldots, b_{m_{i}}^{i}, 0 \leq i \leq r+1$ be non-repetetive sequences with $b_{j}^{i} \in \mathbb{B}$ for all $i=0,1, \ldots, r+1$ and $j=1,2, \ldots, m_{i}$. If $\mathbb{A} \cap \mathbb{B}=\emptyset$ then $S=B^{0}, A_{1, n_{1}}, B^{1}, A_{n_{1}+1, n_{2}}, \ldots, B^{r}, A_{n_{r}+1, m}, B^{r+1}$ is a non-repetitive sequence. 
Proof. Suppose by way of contradiction that $R=r_{1}, \ldots, r_{2 t}$ is a repetitive block of $S$, i.e. $r_{k}=r_{k+t}$ for every $k=1,2, \ldots, t$. $R$ could be a subsequence neither of the sequence $A$ nor of any sequence $B^{i}$ because they are non-repetitive. Hence $R$ consists of some blocks from $A$ and some blocks from several consecutive $B^{i}$ 's, $i \in\{0,1, \ldots, r+1\}$ too. If we delete from $R$ all blocks of $B^{i}, i \in\{0,1, \ldots, r+1\}$, then the obtained sequence $R^{\prime}$ is repetitive. This implies that $A$ is repetitive, which is a contradiction.

The second lemma is an immediate implication of the existence of Thue sequences.

Lemma 4. Every cycle admits a non-repetitive edge-colouring in $\{1,2,3,4\}$ such that exactly one edge is coloured 4.

Proof. Let $C$ be a cycle. Colour an edge $e$ of $C$ with 4. $C \backslash e$ is a path and can be non-repetitively edge-coloured with $\{1,2,3\}$ using a Thue sequence. It is easy to see that such an edge-colouring of $C$ is non-repetitive.

\section{Trees}

An internal vertex of a tree is a vertex, that is not a leaf. An almost even tree is a tree where every internal vertex, except at most one, is of even degree. An edge incident with a leaf is called pending edge.

Note that in a tree any facial trail is in fact a facial path.

Lemma 5. Let $S$ be a subdivision of a graph $K_{1,3}$. Then $\pi_{f}^{\prime}(S)=3$.

Proof. In [4] Breštar et al. showed that if a tree $T$ admits a non-repetitive 3edge-colouring, then there exists a non-repetitive 3-edge-colouring of the tree $T^{\prime}$ obtained from $T$ by subdividing each edge with 12 vertices. Using the above fact recursively on $K_{1,3}$, we obtain that, for each subdivision $S$ of $K_{1,3}, \pi^{\prime}(S)=3$ and consequently $\pi_{f}^{\prime}(S)=3$.

Theorem 6. Let $T$ be an almost even tree. Then $\pi_{f}^{\prime}(T) \leq 3$.

Proof. We prove the result by induction on the number of leaves of $T$.

If $T$ has two leaves then it is a path. Thus, the statement follows from the existence of arbitrary long non-repetitive sequence over three symbols (see [18]). If $T$ has three leaves, then it is a subdivision of $K_{1,3}$ and by Lemma 5 we are done.

Suppose now that $T$ has more than three leaves. Let $R$ be the smallest subtree of $T$ containing all the vertices of degree at least 3 in $T$. Then all the leaves of $R$ have degree at least 3 in $T$. Moreover, because $T$ is almost even, there is a leaf $v$ of $R$ such that $\operatorname{deg}_{T}(v) \geq 4$. Now $T-v$ is a forest of $\operatorname{deg}_{T}(v)$ trees among which at most one is not a path.

If $\operatorname{deg}_{T}(v) \geq 5$, let $P_{0}, P_{1}, P_{2}$ and $P_{3}$ be four paths of $T-v$ which are consecutive in the clockwise order around $v$. Let $T_{1}$ be the tree obtained from $T$ by extending each of the four $P_{i}$ into a path $Q_{i}$ of length equal to the maximal 
length of the $P_{j}, 1 \leq j \leq 4$. Clearly, $T_{1}$ is a supertree of $T$ having the same number of leaves. Let $T_{2}$ be the tree obtained from $T_{1}$ by removing $Q_{1}$ and $Q_{2}$. Then $T_{2}$ has two leaves less than $T_{1}$ and $T$. So, by the induction hypothesis, it admits a facial non-repetitive 3 -edge-colouring. Colouring the edges of $P_{1}$ (resp. $P_{2}$ ) with the same pattern as the edges of $P_{3}$ (resp. $P_{0}$ ), we obtain a facial non-repetitive 3-edge-colouring of $T$.

If $\operatorname{deg}_{T}(v)=4$, the proof is very similar to the one when $\operatorname{deg}_{T}(v) \geq 5$. We consider three paths $P_{1}, P_{2}$ and $P_{3}$ of $T-v$ which are consecutive in the clockwise order around $v$. Then $P_{0}$ is the path of $T$ whose union with $v$ and $P_{1}$ forms a maximal facial path of $T$. The rest of the proof is then identical.

Theorem 7. Let $T$ be a tree and let $K$ be a subtree of $T$ which is almost even. Then there exists a facial non-repetitive 4-edge-colouring of $T$ that uses only 3 colours on the edges of $K$.

Proof. We proceed by induction on the number of internal vertices of odd degree in the tree $T$.

If there are less than two such vertices then $T$ is almost even tree and we are done by Theorem 6 .

Suppose now that $T$ has at least two internal odd degree vertices. Consider a maximal (with respect to inclusion) almost even subtree $S$ of $T$, which contains the tree $K$ as a subgraph. The maximality of $S$ implies that for every vertex $v \in V(S), \operatorname{deg}_{S}(v) \geq \operatorname{deg}_{T}(v)-1$ and that the leaves of $S$ are also the leaves of $T$. In addition, exactly one internal vertex with odd degree in $T$ has all its incident edges in $S$. By Theorem 6 , there exists a facial non-repetitive edge-colouring $\varphi$ of $S$ that uses colours from the set $\{1,2,3\}$.

Let $W$ be the boundary walk of the unique face of $T$ beginning and finishing in the same fixed leaf of the tree $S$. Let $e_{1}, e_{2}, \ldots, e_{k}$ be the edges of $E(T) \backslash E(S)$ having one endvertex in $V(S)$, the indices being taken in the order of appearance on $W$. (Clearly $k$ is the number of internal odd degree vertices of $S$ minus 1.) By maximality of $S$, those edges are pairwise disjoint. For $1 \leq i \leq k$, let $T_{i}$ be the tree of $T \backslash e_{i}$ not intersecting $S$. Each edge $e_{i}$ appears twice in $W$. Let us denote $e_{i, 1}$ and $e_{i, 2}$ (resp. $e_{i, 3}$ and $e_{i, 4}$ ) the edges preceding and succeeding the first (resp. second) appearance of $e_{i}$ along $W$. Clearly $e_{i, 1}$ and $e_{i, 4}$ are in $E(S)$ while $e_{i, 2}$ and $e_{i, 3}$ are not. Note that if the endvertex of $e_{i}$ which is not in $V(S)$ has degree at most 2 , then $e_{i, 2}=e_{i, 3}$. Moreover if $e_{i}$ is a pending edge then $e_{i}=e_{i, 2}=e_{i, 3}$ (in this case $T_{i}$ consists of one vertex only).

Let us now extend the colouring $\varphi$ in the following way: For all $1 \leq i \leq k$, we set $\varphi\left(e_{i}\right)=4$. We then colour the edges of $T_{i}$. Let $K_{i}$ be the subtree of $T_{i}$ induced by the edges of $T_{i}$ that are in a facial path containing $e_{i}$ in $T$. Then $K_{i}$ is either a path or a subdivision of $K_{1,3}$ and thus is almost even. Hence, by the induction hypothesis, there is a facial non-repetitive edge-colouring $\varphi_{i}$ of $T_{i}$ with colours in $\{1,2,3,4\}$ such that no edge of $K_{i}$ is assigned 4 . Free to permute the colours, we may assume that $\varphi_{i}\left(e_{i, 2}\right) \neq \varphi\left(e_{i-1,4}\right)$ and $\varphi_{i}\left(e_{i, 3}\right) \neq \varphi\left(e_{i+1,1}\right)$ (the indices should be taken modulo $k$ ).

Let us show that the union of $\varphi, \varphi_{1}, \ldots, \varphi_{k}$ is a facial non-repetitive edgecolouring of $T$. Let $P$ be a facial path of $T$. Then it contains at most two edges 
of $\left\{e_{1}, \ldots, e_{k}\right\}$.

If $P$ has no edge in this set then it is entirely contained in either $S$ or one of the $T_{i}$ 's. So it is not repetitive since $\varphi_{1}, \ldots, \varphi_{k}$ and $\varphi$ are facially non-repetitive.

If $P$ contains exactly one edge in $\left\{e_{1}, \ldots, e_{k}\right\}$, then this edge is the unique one of $P$ coloured 4 . Hence $P$ is not repetitive.

If $P$ contains two edges in $\left\{e_{1}, \ldots, e_{k}\right\}$, then they are consecutives: there exists $1 \leq i<k$ such that edges $e_{i}$ and $e_{i+1}$ are in $E(P)$. These edges are the only ones in $P$ being coloured with 4 . But since $\varphi\left(e_{i, 4}\right) \neq \varphi_{i+1}\left(e_{i+1,2}\right)$ and $\varphi\left(e_{i, 3}\right) \neq \varphi_{i+1}\left(e_{i+1,1}\right), P$ is not repetitive.

Proposition 8. Let $T$ be the tree depicted in Figure 2. Then $\pi_{f}^{\prime}(T)=4$.

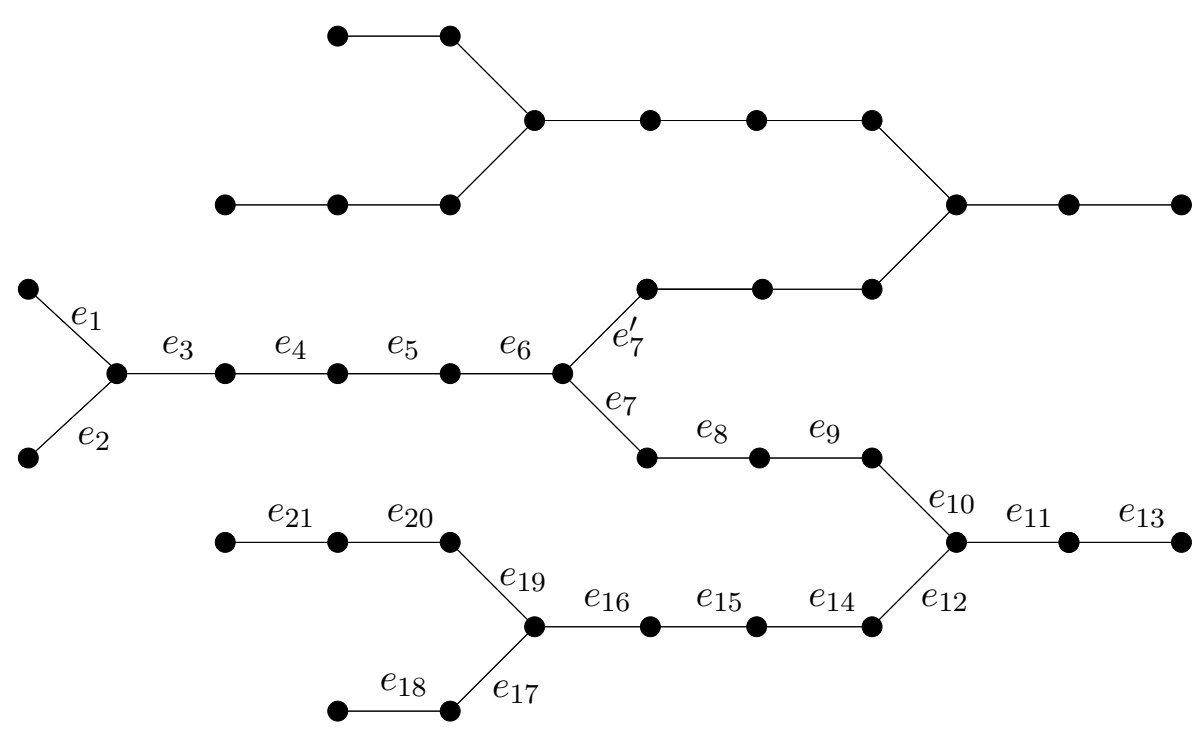

Figure 2: A tree with facial Thue chromatic index 4

Proof. Let us show that $T$ has no facial non-repetitive edge-colouring with 3 colours. Suppose by way of contradiction that such an edge-colouring $c$ exists with colour set $\{1,2,3\}$. Without loss of generality, we may assume that $c\left(e_{i}\right)=$ $i$ for $1 \leq i \leq 3$. Moreover, by symmetry, we may assume that $c\left(e_{4}\right)=2$. Then $c\left(e_{5}\right)=1$ otherwise $\left(e_{2}, e_{3}, e_{4}, e_{5}\right)$ would be repetitive. We have $c\left(e_{6}\right) \neq 2$ otherwise one edge $f$ of $\left\{e_{7}, e_{7}^{\prime}\right\}$ is coloured 1 and $\left(e_{4}, e_{5}, e_{6}, f\right)$ is repetitive. Thus $c\left(e_{6}\right)=3$. Now $c\left(e_{7}^{\prime}\right)=1$ otherwise $\left(e_{1}, e_{3}, e_{4}, e_{5}, e_{6}, e_{7}^{\prime}\right)$ would be repetitive. So $c\left(e_{7}\right)=2$. Moreover because $\left(e_{3}, e_{4}, e_{5}, e_{6}, e_{7}, e_{8}\right)$ is not repetitive, $c\left(e_{8}\right)=3$ and because $\left(e_{6}, e_{7}, e_{8}, e_{9}\right)$ is not repetitive, $c\left(e_{9}\right)=1$. With similar arguments, we deduce that $c\left(e_{10}\right)=2, c\left(e_{11}\right)=1, c\left(e_{12}\right)=3, c\left(e_{13}\right)=3, c\left(e_{14}\right)=2$, $c\left(e_{15}\right)=1, c\left(e_{16}\right)=3, c\left(e_{17}\right)=1, c\left(e_{18}\right)=2, c\left(e_{19}\right)=2, c\left(e_{20}\right)=3$ and $c\left(e_{21}\right)=1$. But then $\left(e_{2}, e_{3}, e_{4}, e_{5}, e_{6}, e_{7}, e_{8}, e_{9}, e_{10}, e_{12}, e_{14}, e_{15}, e_{16}, e_{19}, e_{20}, e_{21}\right)$ is repetitive. 
Denote by $\pi_{f}^{\prime}(\mathcal{T})$ the maximum of $\pi_{f}^{\prime}(T)$ over all trees $T$. Theorem 7 says that $\pi_{f}^{\prime}(\mathcal{T}) \leq 4$. Together with Proposition 8 , this gives

Theorem 9. $\pi_{f}^{\prime}(\mathcal{T})=4$.

\section{General upper bounds}

The Lemma 10 appears in [3], p. 258. For the sake of completeness we provide a simple proof of it. We need the lemma in the following formulation.

Lemma 10. Let $G$ be a connected plane graph and $T^{*}$ be a spanning tree of its dual $G^{*}$. Let $T$ be a subgraph of $G$ with edge set $E(T)$ associated to the edge set of $T^{*}$. Then $G \backslash E(T)$ is a tree.

Proof. Clearly $|E(T)|=\left|E\left(T^{*}\right)\right|=\left|V\left(G^{*}\right)\right|-1=|F(G)|-1$. With Euler's Formula $(|E(G)|+2=|V(G)|+|F(G)|)$ it implies $|E(G)|-|E(T)|=|V(G)|-1$. It is enough to show that $G \backslash E(T)$ is connected. If not, then $E(T)$ contains an edge cut set which corresponds to a cycle in $T^{*}$ - a contradiction.

Therefore $G \backslash E(T)$ is connected on $|V(G)|-1$ edges and thus $G \backslash E(T)$ is a tree, see [3].

Theorem 11. Let $G$ be a connected plane graph. Then $\pi_{f}^{\prime}(G) \leq 8$.

Proof. Denote by $T^{*}$ a spanning tree of $G^{*}$. Let $T$ be the subgraph of $G$ induced by the edges associated to the edges of $T^{*}$. By Lemma 10, $G \backslash E(T)=T_{0}$ is a tree and hence there is a facial non-repetitive edge-colouring $\varphi\left(T_{0}\right)$ using colours from $\{1,2,3,4\}$.

Let us colour the edges of $E(T)$ with the four new colors $\{5,6,7,8\}$. Let us root the tree $T^{*}$ in some of its leaf $f_{0}$. For every face $f \neq f_{0}$ of $G$, we denote by $e_{f}$ the edge shared by $f$ and its parent in the rooted tree $T^{*}$. We colour the (uncoloured) edges of the faces of $G$ successively from the root $f_{0}$ to the leaves of $T^{*}$. First, we assign to the unique uncoloured edge incident with $f_{0}$ the colour 5. Then at each step, we shall colour the uncoloured edges of a face $f$ with colours in $\{5,6,7,8\} \backslash c_{f}$, where $c_{f}$ is a colour of the edge $e_{f}$ received by $e_{f}$ when colouring the parent of $f$. So far, all the coloured edges of $f$ are those in $E\left(T_{0}\right)$ which are coloured by $\varphi$ except $e_{f}$ which is coloured with $c_{f} \in\{5,6,7,8\}$. Let $e_{1}, e_{2}, \ldots, e_{k}$ be uncoloured edges incident with $f$ in clokwise order around $f$ starting at $e_{f}$. (Note that no edge from the set $\left\{e_{1}, e_{2}, \ldots, e_{k}\right\}$ is a bridge of $G$. Otherwise if, say, $e_{i}$ is a bridge then the associated edge $e_{i}^{*}$ in $G^{*}$ is a loop. But no loop can appear in $T^{*}$.) We colour the $e_{i}$ 's non-repetitively with three colours of $\{5,6,7,8\} \backslash\left\{c_{f}\right\}$. Doing so, no trail on the boundary of $f$ is a repetition because the colour of $e_{f}$ appears only once around $f$ and the colouring of the edges of $f \backslash e_{f}$ is non-repetitive by Lemma 3 .

Theorem 12. Let $G$ be a simple 3-connected plane graph. Then $\pi_{f}^{\prime}(G) \leq 7$. 
Proof. The dual graph $G^{*}$ of the graph $G$ is a simple 3-connected plane graph (see [3], p. 258). By Barnette's Theorem [2] $G^{*}$ contains a spanning tree $T^{*}$ of maximum degree $\Delta\left(T^{*}\right) \leq 3$. To prove that there is a facial non-repetitive colouring $\varphi$ of $G$ with 7 colours we proceed analogously as in the proof of Theorem 11. First we colour the edges of $G \backslash E(T)$ with colours from the set $\{1,2,3,4\}$ analogously to the proof of Theorem 7. After the colouring $\varphi$, each face has all its edges coloured except at most three. To colour the remaining edges of $T$ it is sufficient to use three new colours from $\{5,6,7\}$ in the same way as in the proof of Theorem 11. The details are left to the reader.

Motivated by the proof of Theorem 12 one can prove the following

Theorem 13. Let $G$ be a connected plane graph and let its dual contains a Hamilton path $H^{*}$. Then $\pi_{f}^{\prime}(G) \leq 6$.

Proof. To prove that there is a facial non-repetitive colouring $\varphi$ of $G$ with 6 colours we proceed analogously as in the proof of Theorem 11. First we colour the edges of $G \backslash E(H)$ with colours from the set $\{1,2,3,4\}$ non-repetitively as in the proof of Theorem 7. Here $E(H)$ is the set of edges corresponding to those of $H^{*}$ in $G^{*}$. After this colouring, each face has all its edges coloured except at most two. To colour the remaining edges of $H$ it is sufficient to use two new colours 5 and 6 in the same way as in the proof of Theorem 11. The details are left to the reader.

A Halin graph is a plane graph which is an union of a tree $T$ of order $p \geq 3$ having no vertices of degree 2 and a cycle $C$ connecting the leaves of $T$ in a cyclic order determining by a plane embedding of $T$. It is easy to see that the dual of any Halin graph contains a Hamilton path. Applying Theorem 13 we get the following.

Corollary 14. Let $G$ be a Halin graph. Then $\pi_{f}^{\prime}(G) \leq 6$.

\section{$5 \quad$ Plane hamiltonian graphs}

A Hamilton cycle in a graph $G$ is a cycle containing all the vertices. A graph $G$ containing a Hamilton cycle is said to be hamiltonian. The aim of this section is to give better upper bounds on the facial Thue chromatic index of plane graph, provided that it is hamiltonian.

Let $G$ be a plane hamiltonian graph and $H$ a Hamilton cycle in $G$. We denote by $G_{1}$ (resp. $G_{2}$ ) the subgraph of $G$ induced by $H$ and the edges of $G$ inside (resp. outside) of $H$. Evidently $G_{i}, i=1,2$, is a 2 -connected outerplanar graph. Recall that a plane graph is outerplanar if all its vertices are incident to the outer face.

The weak dual $G^{w}$ of a planar graph $G$ is the graph obtained from $G^{*}$ by deleting the vertex corresponding to the outer face of $G$ (and all the edges incident with it). Fleischner et al. [8] showed that if $G$ is outerplanar then $G^{w}$ is a forest and that it is a tree if $G$ is 2 -connected. For $i=1,2$, we denote $T_{i}$ the weak dual of $G_{i}$. The edges of $G_{i}$ not on $H$ are called chords. 
Theorem 15. Let $G$ be a hamiltonian plane graph and $H$ a Hamilton cycle of $G$. Then $\pi_{f}^{\prime}(G) \leq 7$.

Moreover, if $\max \left\{\Delta\left(T_{1}\right), \Delta\left(T_{2}\right)\right\} \leq 4$ then $\pi_{f}^{\prime}(G) \leq 6$

and if $\max \left\{\Delta\left(T_{1}\right), \Delta\left(T_{2}\right)\right\} \leq 2$ then $\pi_{f}^{\prime}(G) \leq 5$.

Proof. Let us first colour non-repetitively the edges of $H$ with $\{1,2,3,4\}$ so that exactly one edge $e_{0}$ is coloured with 4 . This is possible according to Lemma 4.

Next we colour the chords of $G_{1}$. To do so we root the tree $T_{1}$ in $f_{0}$ - the face of $G_{1}$ incident to $e_{0}$. For every face $f$, let $e_{f}$ be the edge shared by $f$ and its parent if $f \neq f_{0}$ or be $e_{0}$ if $f=f_{0}$.

We now colour the chords of the faces of $G_{1}$ successively form the root $f_{0}$ to the leaves of $T_{1}$. At each step, we shall colour the uncoloured chords of a face $f$ with colours in $\{4,5,6,7\}$. So far, all the coloured edges of $f$ are those in $H \backslash\left\{e_{0}\right\}$ which are coloured in $\{1,2,3\}$ except $e_{f}$ which is coloured $c_{f} \in\{4,5,6,7\}$. Let $e_{1}, e_{2}, \ldots, e_{k}$ be uncoloured chords incident to $f$ in clokwise order around $f$ starting at $e_{f}$. We colour the $e_{i}$ with a non-repetitive sequence with the three colour of $\{4,5,6,7\} \backslash\left\{c_{f}\right\}$. Doing so, no trail on the boundary of $f$ is a repetition because the colour of $e_{f}$ appears only once around $f$ and $f \backslash e_{f}$ is non-repetitive by Lemma 3.

We proceed analogously to colour the chords of $G_{2}$ to obtain a facial nonrepetitive edge-colouring of $G$.

If $\max \left\{\Delta\left(T_{1}\right), \Delta\left(T_{2}\right)\right\}$ is at most 4 (resp. 2 ), we proceed in a similar way as above. But at each step we want the face $f$ of $G_{1}$ or $G_{2}$ in consideration to have at most 3 (resp. 1) non-coloured edges. This will guarantee that 2 colours (resp. 1 colour) are enough for a non-repetitive colouring of the uncoloured chords. So the set $\{4,5,6\}$, (resp. $\{4,5\})$ is enough to colour all the chords. If $|V(G)| \notin\{5,7,9,10,14,17\}$ then colouring non-repetitively the outer face with $\{1,2,3\}$ and taking $f_{1}$ to be a leaf of $T_{1}$ (or $T_{2}$ ), we have the desired property. The cases when $|V(G)| \in\{5,7,9,10,14,17\}$ are left to reader.

An almost identical proof in which the Hamilton cycle $H$ is replaced by the facial walk of the outer face yields the result for 2-edge-connected outerplanar graphs in which every inner face has a chord.

The bound of Theorem 15 applies for 2-connected outerplanar graphs.

Theorem 16. Let $G$ be a 2-connected outerplanar graph. Then $\pi_{f}^{\prime}(G) \leq 7$. Moreover denoting by $T$ be the weak dual of $G$ the following hold:

(i) if $G$ is a cycle and $|V(G)|=2$, then $\pi_{f}^{\prime}(G)=2$;

(ii) if $G$ is a cycle and $|V(G)| \notin\{2,5,7,9,10,14,17\}$, then $\pi_{f}^{\prime}(G)=3$;

(iii) if $G$ is a cycle and $|V(G)| \in\{5,7,9,10,14,17\}$, then $\pi_{f}^{\prime}(G)=4$;

(iv) if $\Delta(T) \leq 2$, then $\pi_{f}^{\prime}(G) \leq 5$;

(v) if $\Delta(T) \leq 4$, then $\pi_{f}^{\prime}(G) \leq 6$. 


\section{Discussion}

In this paper, we gave upper bounds for the facial Thue chromatic index of various classes of plane graphs. However most of these upper bounds are certainly not tight. Hence, for each of these classes $\mathcal{C}$, it would be nice to find its facial Thue chromatic index $\pi_{f}^{\prime}(\mathcal{C})=\max \left\{\pi_{f}^{\prime}(G), G \in \mathcal{C}\right\}$ especially for the class $\mathcal{P}$ of plane graphs.

Problem 17. What is the value of $\pi_{f}^{\prime}(\mathcal{P})$ ? In other words, what is the smallest integer $k$ such that $\pi_{f}^{\prime}(G) \leq k$ for all plane graphs $G$ ?

We believe that the following is true

Conjecture 18. Let $G$ be a 3 -connected plane graph. Then $\pi_{f}^{\prime}(G) \leq 6$.

There are many generalisations of non-repetitive sequences and for most of them it also make sense to study their graph theoretical variants. In principle, any property of sequences can be translated into a property of graphs, via coloured trails. In particular, one may take any avoidable pattern (see [7]) and study its behaviour on graphs. In another direction, one may look at vertexcolouring instead of edge-colouring. The Thue chromatic number of $G$, denoted $\pi(G)$, is the minimum number of colours of a non-repetitive vertex-colouring of $G$. Several papers study non-repetitive colourings, see e.g. $[1,4,5,10,11,12$, 13, 14]. A major conjecture in this area is due to Grytczuk [11].

Conjecture 19 (Grytczuk [11]). There exists an absolute constant $k$ such that any planar graph has a non-repetitive vertex $k$-colouring.

Certainly, it is equally interesting to study the facial version of all the abovementionned variants. In particular, showing that the facial Thue chromatic number of a plane graph is bounded would be a first step towards Conjecture 19.

\section{Acknowledgement}

We are grateful to Igor Fabrici for his stimulating discussion concerning precise value of the facial Thue chromatic index for trees.

This work was supported by the Slovak Science and Technology Assistance Agency under the contract No APVV-0007-07 and partially supported by the european project FET - AEOLUS.

\section{References}

[1] N. Alon, J. Grytczuk, M. Haluszcak, O. Riordan, Non-repetitive colourings of graphs, Random. Struct. Algor. 21 (2002), 336-346.

[2] D. W. Barnette, Trees in polyhedral graphs, Canad. J. Math. 18 (1966), 731-736. 
[3] J. A. Bondy, U. S. R. Murty, Graph Theory, Springer, 2008.

[4] B. Breštar, J. Grytzuk, S. Klavžar, S. Niwczyk, I. Peterin, Non-repetitive colorings of trees, Discrete Math. 307 (2007), 163-172.

[5] J. D. Currie, Open problems in pattern avoidance, The American Mathematical Monthly 100 (1993), 790-793.

[6] J.D. Currie, There are ternary circular square-free words of length $n$ for $n \geq 18$, Electron. J. Combin. 9 (2002), \#N10.

[7] J.D. Currie, Pattern avoidance: themes and variations, Theoret. Comput. Sci. 339 (2005), 7-18.

[8] H.J. Fleischner, D.P.Geller, F. Harary, Outerplanar graphs and weak duals, J. Indian Math Soc. 38 (1974), 215-219.

[9] R. P. Gupta, The chromatic index and the degree of a graph, Not. Amer. Math. Soc. 13 (1966), 719.

[10] J. Grytczuk, W. Śliwa, Non-repetitive colorings of infinite sets, Discrete Math. 265 (2003), 365-373.

[11] J. Grytczuk, Thue type problems of graphs, points and numbers, Discrete Math. 308 (2008), 4419-4429.

[12] J. Grytczuk, Pattern avoidance on graphs, Discrete Math. 307 (2007), 13411346 .

[13] A. Kündgen, M.J. Pelsmajer, Non-repetitive colorings of graphs of bounded tree-width, Discrete Math. 308 (2008), 4473-4478.

[14] N. Rampersad, A note on non-repetitive colourings of planar graphs, arXiv:math/0307365 v 1, (2003), 1-3.

[15] N. Robertson, D.P. Sanders, P. Seymour and R. Thomas, The four-color theorem, J. Combin. Theory Ser. B 70(1997), 2-44.

[16] C. E. Shannon. A theorem on colouring lines of a network, J. Math. Phys. 28 (1949), 148-151.

[17] P. G. Tait, Note on the Theorem in geometry of position, Trans. Roi. SOC. Edinburgh 29 (1880), 657-660.

[18] A. Thue, Über unendliche Zeichenreichen, Norske Vid. Selsk. Skr., I Mat. Nat. Kl., Christiana 7 (1906), 1-22 (In German).

[19] V. G. Vizing, On an estimate of the chromatic class of a p-graph, Metody Diskret. Analyz. 3 (1964), 25-30.

[20] R. A. Wilson, Graphs, colourings and the four-colour theorem, Oxford University Press, New York (2002) 